$$
\begin{aligned}
i_{i} \gamma_{2} & =m_{i}^{2}+m_{i}\left(\gamma_{1}+2-\alpha_{i}^{2}\right)+n_{i}\left(\gamma_{1}+\alpha_{i}^{2}\right)+\gamma_{1} \\
h_{i} k^{2}\left(1-k^{2}\right) & =\left(\alpha_{i}^{2}-k^{2}\right)\left(k^{4}+2 k^{2} \gamma_{1}+r^{2}\right) .
\end{aligned}
$$

This permits us to write formulas $416.00,417.00,437.00$ and 438.00 with no explicit. appearances of $m_{1}$ or $m_{2}$. For example, 416.00 becomes:

$$
\begin{aligned}
416.00^{\prime}: a \Pi\left(\alpha^{2}\right)+a \Pi\left(\alpha^{2}\right)= & \frac{2}{s_{1} t_{2}-s_{2} t_{1}}\left\{\left[a_{1}\left(t_{1}-t_{2}\right)+b_{1}\left(s_{1}-s_{2}\right)\right] K\right. \\
& \left.+n_{2}\left(a_{1} t_{1}+b_{1} s_{1}\right) \Pi\left(\alpha_{2}^{2}\right)-n_{1}\left(a_{1} t_{2}+b_{1} s_{2}\right) \Pi\left(\alpha_{1}^{2}\right)\right\}
\end{aligned}
$$

A similar simplification is possible in the special case when $m_{1}=0=\alpha_{1}^{2}$. In this case we multiply the second equation of (3) by $m_{2}$ as above, and multiply equation (8) by $r^{2}$ to obtain

$$
s_{1} \Pi_{1}+t_{1} \Pi_{2}=-k^{2} F(\phi)+r \tanh ^{-1} \frac{r \cos \phi \sin \phi}{\Delta}
$$

where

$$
\begin{aligned}
s_{1} & =r^{2}-k^{2} \\
t_{1} \gamma_{2} & =2 r^{2}+r^{2} \gamma_{1}+k^{2}
\end{aligned}
$$

$\left(n_{2}, s_{2}\right.$ and $t_{2}$ are defined as in $\left(7^{\prime}\right)$.) This permits us to write formulas 418.00, $419.00,439.00$, and 440.00 with no explicit appearances of $m_{2}$ or $r_{2}$ (But note that the two occurrences of $r$ in formulas 439.00 and 440.00 are preserved.) For example, 439.00 (with the sign changes mentioned in Section 4 incorporated) becomes

$$
\begin{aligned}
439.00^{\prime}: a \Pi\left(u_{1}, \alpha^{2}\right)+\bar{a} \Pi\left(u_{1}, \bar{\alpha}^{2}\right) & =\frac{2}{s_{2} t_{1}-t_{2} s_{1}}\left\{\left[a_{1}\left(k^{2} t_{2}-t_{1}\right)+b_{1}\left(k^{2} s_{2}-s_{1}\right)\right] u_{1}\right. \\
& -n_{2}\left(a_{1} t_{1}+b_{1} s_{1}\right) \Pi\left(u_{1}, \alpha_{2}{ }^{2}\right)+\left(a_{1} t_{1}+b_{1} s_{1}\right) \tau^{2} \\
& \left.-r\left(a_{1} t_{2}+b_{1} s_{2}\right) \tanh ^{-1} \frac{r \cos \phi \sin \phi}{\Delta}\right\} .
\end{aligned}
$$

The Rand Corporation

Santa Monica, California

1. G. J. HoüzL, Recueil de Formules et de Tables Vumériques, Gauthier-Villars. Paris, 1901

2. Paul F. BYrd \& Morris D. FriedMan, Handbook of Elliptic Integrals for Engineers and Physicists, Springer-Verlag, Berlin, 1954.

\title{
The Numerical Evaluation of the Eighteenth Perfect Number
}

\section{By D. Scheffler and R. Ondrejka}

On November 17, 1959 the IBM 709 installation at the National Aviation Facilities Experimental Center in Atlantic City, New Jersey computed the largest known perfect number, corresponding to the eighteenth Mersenne prime [1]. The result was checked by recomputation one week later. Running time for this cal-

Received December 2, 1959. 
culation was approximately five minutes. This perfect number has 6433 proper divisors, and its decimal representation is as follows:

\begin{tabular}{|c|c|c|c|c|c|c|c|c|c|}
\hline & & $3: 3$ & 5708.3 & 21319 & 86724 & 43701 & 08772 & 80 & 84 \\
\hline 1380 & 3499 & 87972 & 54.549 & 96241 & $\tilde{5} 7348$ & 21584 & 50444 & 04288 & 20708 \\
\hline & 7699 & 0.3884 & 59.5.35 & 77426 & 08498 & 85573 & 69475 & 99061 & 38 \\
\hline & & $301: 30$ & 80704 & 76236 & 5.5942 & 23617 & 48505 & $0910 \varepsilon$ & 378 \\
\hline & 6642 & 32548 & 24947 & 61473 & 19657 & 90746 & 56099 & $9186 C$ & 076 \\
\hline & 18166 & 02944 & 699121 & 7787:3 & 79658 & 22199 & 90166 & & $\mathbf{0}$ \\
\hline 7502 & 23592 & 23201 & 84998 & 56361 & 44 & 18 & 54020 & De & 01 \\
\hline & 97727 & 0848: & 94647 & 43635 & 03718 & 15002 & 84915 & & \\
\hline & & $29: 560$ & 72060 & 01347 & 49556 & 17851 & 48168 & 01859 & 8855 \\
\hline 60 & 92248 & 41817 & 8 & & & & & & \\
\hline 7 & 106667 & $3992: 35$ & 10 & 74 & 0 & & 6 & 34 & 0900 \\
\hline & $7026 !)$ & 75942 & $410: 31$ & 95 & 98 & 71 & 00 & 46 & 74673 \\
\hline 94 & 44715 & $(i 25)(60)$ & 0.5717 & 96578 & 81 & & 21029 & & 29975 \\
\hline & 40515 & 17095 & 61679 & 51095 & 45364 & & & 66 & \\
\hline 60 & 01177 & $019: 32$ & 74226 & & & & & 5 & 54 \\
\hline 510 & $\dot{4}$ & $5(6745$ & 9.3 & 01 & 0 & & & & \\
\hline & $16 i 3$ & $15.5 ! 98$ & 24715 & 649 & 19 & 16 & & & 95091 \\
\hline 98074 & 1!) & 34095 & 80454 & & & & & & \\
\hline & & Of(i)i45) & 13775 & & 53 & & & & 3 \\
\hline & .) & 11 & 38 & & & & & & \\
\hline 72 & 1160 & 91179 & 78404 & 56 & 49 & & & & \\
\hline 44495 & 12:38:3 & $771: 39$ & 620 & & & & & & \\
\hline & 56156 & 19951 & & & & & & & \\
\hline 7 & 595.56 & 0 & 59 & & & & & 4 & 07 \\
\hline & 435521 & $125+2$ & 19 & 4 & $94^{\prime}$ & & & & 27467 \\
\hline $4(63329$ & 85210 & 42107 & $55: 317$ & 81 & & & & & 1 \\
\hline 65 & $71: 348$ & $4: 3880$ & 4: & & 54 & & & & \\
\hline 59113 & $126(i 2)$ & $32+22$ & 00 & & & & & & \\
\hline 67362 & $1928: 3$ & 02111 & 92876 & 17896 & 14688 & 558 & 60 & 04 & \\
\hline & & & & & & & & & \\
\hline & 94601 & 56797 & 8.5. & & & & & & \\
\hline 831 & $85+68$ & 240933 & & & & & & & 1 \\
\hline & 00881 & $587+7$ & & & 72 & & & & 9121 \\
\hline & 62611 & 21386 & & & 39 & & & & 1786 \\
\hline & $74: 39: 3$ & 03504 & $90 \div 26$ & 03882 & 47 & & & & \\
\hline 692 & 597 & 37781 & 93050 & 34874 & & & & & \\
\hline $19 !$ & & $385 \overline{5} 7$ & & & 60647 & & & & \\
\hline & & 19702 & 68 & & 64 & & & 46345 & \\
\hline & & & 7 & 911 & & & 88 & 26285 & \\
\hline
\end{tabular}

Computation Branch

National Aviation Facilities Experimental Center

Atlantic City, N. J.

1. H. Riesel, "A new Mersenne prime." MTAC, v. 12, 1958, p. 60. 